\title{
Substance Use Disorder and Telemedicine: Opportunity and Concern for the Future
}

\author{
Mubeen Shakir, MD, MPP, MSc (1) and Sarah Wakeman, MD
}

Department of Medicine, Massachusetts General Hospital, Boston, MA, USA.

$\mathrm{J}$ Gen Intern Med 36(9):2823-4

DOI: $10.1007 / \mathrm{s} 11606-020-06299-8$

(c) Society of General Internal Medicine 2020

$\mathrm{T}$ he COVID-19 pandemic has forced health systems across the USA to adapt, innovating systems of care for the protection of patients and public health. The most apparent transformation is the sudden shift to telemedicine across specialties. Prior to the crisis, nearly 13,000 Medicare beneficiaries received telemedicine care each week; in the last week of April alone nearly 2 million patients received telemedicine services. This rapid expansion in telemedicine services has included care for patients with substance use disorder (SUD) where access issues have long prevented adequate care delivery. ${ }^{1}$

There are clear benefits to telemedicine in delivering care for patients with SUD. In addition to reducing transmission risk of COVID-19, telemedicine also has the potential to increase access. A phone visit reduces the barriers of coming to the office for a patient who might be reliant on public transportation, may need extra time off for work, or might need to find child care. Importantly, for opioid use disorder treatment in particular, telemedicine may offer access to medication treatment for patients who have not previously been able to initiate care in a brick and mortar clinic. New policy changes now allow for the initiation buprenorphine over the phone; this with aligned reimbursement could increase access to lifesaving care even during periods of lock down. ${ }^{2}$ The move to telemedicine presents an opportunity to positively impact the lives of patients with SUD, but its implementation must be deliberate to not exacerbate the structural inequities that already plague the care of these patients.

Given the overall increased risk of mortality among those with SUD relative to other patients, especially those with a history of non-fatal overdose, it is imperative that a shift to telemedicine does not impact the quality of care for these patients. Preliminary data already indicates that overdose

Received September 10, 2020

Accepted October 5, 2020

Published online October 19, 2020 deaths have skyrocketed since the start of the pandemic, with some areas recording a near doubling of deaths. ${ }^{3}$

While the benefits cannot be overstated, concerns have already been raised about the potential of the shift to telemedicine to exacerbate disparities already driven by racism and poverty. Patients with SUD likely also face more significant telemedicine access issues than other patient populations due to ongoing structural inequities. SUD disproportionately affects patients experiencing homelessness and poverty. ${ }^{4}$ The overdose crisis is also dramatically impacting Black and Latinx patients, who experienced the highest rates of increase in overdose deaths from 2013 to 2017, largely driven by illicitly manufactured fentanyl..$^{5}$ And yet, because of racist drug policy, Black and Latinx individuals are disproportionately criminalized and have lower access to and retention in effective SUD care.

Patients with SUD who experience poverty or homelessness may not have consistent cellphone service or Wi-Fi access to conduct a phone or video visit. These issues are further compounded by privacy issues. Effective SUD treatment requires confidentiality and trust, both of which are not guaranteed if the patient cannot find a safe and secure place to talk.

Regulatory barriers remain a significant challenge to care for patients with SUD, even with the adaptations since the start of the pandemic. Though there is new flexibility by payers and regulations to allow for initiation of buprenorphine over the phone; methadone, another lifesaving medication for opioid use disorder (MOUD), continues to be highly regulated and requires an in-person initial visit. Although regulation changes allowed under the public health emergency of COVID allow more access to take-home methadone doses in some cases, there is heterogeneity in utilization of this new flexibility. Limiting take-home medication increases the risk patients may face in getting to an opioid treatment program to receive daily methadone. Daily attendance requirements may also prevent some individuals from seeking methadone treatment at all.

Effective treatment for patients with SUD should be individualized. A range of care team members, including providers, case managers, recovery coaches, office-based addiction treatment nurses, and behavioral health clinicians may be involved in the coordination and pooling of resources and frequent touches for a patient. Flexibility and patient choice in how these different touchpoints occur is ideal to engage 
patients in ongoing care. Telemedicine done the wrong way could limit this flexibility and individualization of care. In addition, appropriate harm reduction services are critical, and access to naloxone and sterile use equipment will be challenging in the COVID era. Lastly, some patients simply may not want to engage with care through telemedicine.

With these limitations and challenges in mind, there are guiding principles that providers and health systems must use to best care for our marginalized patients with SUD. First, any improvement of access to care that comes with telemedicine, and particularly access to MOUD, should be viewed as positive. However, we must ensure that this shift does not exacerbate racism and structural inequality that already plagues our system. To this end, patients need safe and secure access to telehealth. If we find an increasing need for telemedicine for the safety of the public, we must find a way to care for our most marginalized patients through providing phones and paying for phone bills or Wi-Fi.

Second, further regulatory barriers around MOUD must be lifted to properly care for patients with SUD. Initiation of methadone over the phone should be permissible and reimbursable, and increased take-home medication must be allowed to keep these patients safe during lockdowns without sacrificing access. Counseling requirements have never been based on evidence; in the context of a pandemic, these barriers not only limit access to lifesaving medical therapies but also increase risks for providers and patients alike.

Finally, systems of care must be designed with input, involvement, and guidance from the patients we serve. If patients with SUD do not engage in telemedicine, we must ask them how they prefer to receive care, including improved access to low threshold clinical models and continued provision of harm reduction resources. When patients with SUD do interact physically with the health system, be it through inpatient hospitalizations or ED visits, these opportunities to engage in care should be utilized to ensure that a lack of physical connection does not increase harm.

As we continue to see the effects of initial lockdowns on health outcomes across the disease spectrum, health systems must be agile in caring for patients at the highest risk of suffering and mortality as access is threatened. Focus on our care delivery for patients with SUD, who are deeply marginalized, must be a priority given the risk of death and disparity for these patients even in times of adequate access.

Corresponding Author: Mubeen Shakir, MD, MPP, MSc; Department of Medicine, Massachusetts General Hospital, Boston, MA, USA (e-mail: mshakir@mgh.harvard.edu).

\section{REFERENCES}

1. Verma S. Early impact of CMS expansion of medicare telehealth during COVID-19. Health Affairs Blog. 2020.

2. Walley AY, Alperen JK, Cheng DM et al. Office-Based Management of Opioid Dependence with Buprenorphine: Clinical Practices and Barriers. J Gen Intern Med. 2008;23:1393-1398.

3. Wan W, Long H. 'Cries for help': Drug Overdoses are soaring during the coronavirus pandemic. Wash Post. 2020.

4. Midboe AM, Byrne T, Smelson D, Jasuja G, McInnes K, Troszak LK. The Opioid Epidemic In Veterans Who Were Homeless or Unstably Housed. Health Aff. 2019;38(8): 1289-1297.

5. Agency for Healthcare Research and Quality. Blacks Experiencing fastrising rates of overdose deaths involving synthetic opioids other than methadone. 2020. Publication No. 20-0032-1

Publisher's Note: Springer Nature remains neutral with regard to jurisdictional claims in published maps and institutional affiliations. 